\title{
On exact symmetries and massless vectors in holographic flows and other flux vacua
}

\author{
Massimo Bianchi ${ }^{a, b}$ and Wayne de Paula ${ }^{a, c}$ \\ a Dipartimento di Fisica, Università di Roma "Tor Vergata", \\ I.N.F.N. Sezione di Roma "Tor Vergata", \\ Via della Ricerca Scientifica, 00133 Roma, Italy \\ ${ }^{b}$ Physics Department, Theory Unit, CERN, \\ CH 1211, Geneva 23, Switzerland \\ ${ }^{c}$ Departamento de Fisica, Instituto Tecnologico de Aeronautica, \\ 12228-900 Sao Jose dos Campos, SP, Brazil \\ E-mail: Massimo.Bianchi@roma2.infn.it, lspwayne@hotmail.com
}

Abstract: We analyze the isometries of Type IIB flux vacua based on the PapadopolousTseytlin ansatz and identify the related massless bulk vector fields. To this end we devise a general ansatz, valid in any flux compactification, for the fluctuations of the metric and $p$-forms that diagonalizes the coupled equations. We then illustrate the procedure in the simple case of holographic flows driven by the RR 3-form flux only. Specifically we study the fate of the isometries of the Maldacena-Nuñez solution associated to wrapped D5-branes.

KEYWORDS: Flux compactifications, Gauge-gravity correspondence, AdS-CFT Correspondence, Superstring Vacua

ARXIV EPRINT: 1003.2536 


\section{Contents}

1 Introduction $\quad 1$

2 Vector fields in holographic renormalization 2

3 Field equations and PT ansatz 3

3.1 PT ansatz 4

3.2 Killing vectors 5

$\begin{array}{lll}3.3 & \text { Discrete symmetries and closed subsectors } & 7\end{array}$

4 Exact symmetries and massless vectors $\quad 7$

$4.1 \Omega$ invariant massless vectors (i.e. $F_{5}=0$ and $H_{3}=0$ ) 9

$\begin{array}{lll}\text { 4.1.1 Dilaton equation (consistency check) } & 10\end{array}$

$\begin{array}{lll}4.1 .2 & 3 \text {-form equation } & 10\end{array}$

$\begin{array}{lll}4.1 .3 & \text { Einstein equations } & 12\end{array}$

$\begin{array}{lll}4.2 & \text { One-forms associated to Killing vectors } & 13\end{array}$

$\begin{array}{lll}4.3 & \text { Scalar products and gauge kinetic functions } & 15\end{array}$

5 Massless vectors in MN background $\quad 16$

$\begin{array}{llr}5.1 \text { MN Solution } & 16\end{array}$

$\begin{array}{ll}5.2 \text { Spectrum of massless vector harmonics } & 17\end{array}$

$\begin{array}{llr}6 & \text { Conclusions and summary } & 18\end{array}$

\section{Introduction}

Flux vacua [1] find many interesting applications in String Theory, ranging from holographic flows dual to non (super)conformal boundary theories [2-8] to moduli stabilization in phenomenologically viable models with open and unoriented strings and otherwise [9]. More recently unexpected implications of the AdS/CFT correspondence [10-13] in condensed matter physics $[14,15]$, astrophysics $[16,17]$ and gravity at a Lifschitz point [18] have attracted a lot of attention.

Due to the presence of fluxes, fields belonging to different sectors tend to mix with one another, compatibly with the residual (super)symmetry. Resolving the mixing and finding the spectrum of excitations is extremely laborious [19-21] as witnessed by the enormous effort needed to accomplish the task for the metric and active ${ }^{1}$ scalar modes in holographic flows described by the Papadopoulos-Tseytlin (PT) ansatz [22, 23, 26, 27]. Our aim is to

\footnotetext{
${ }^{1} \mathrm{By}$ active we indicate those scalars with a non trivial profile in the background. All the other (pseudo)scalars are said to be inert.
} 
extend this kind of analysis to the vector sector [19-21, 28, 29]. To this end, we will start by studying the fate of bulk symmetries of the Type IIB supergravity solutions. Although we will mostly adopt a 10-d perspective, we will also present the 5 -d viewpoint, that has a more direct applicability in Holographic Renormalization [20, 21, 30-36] and Holographic QCD [37-41].

Symmetries can be divided into three classes

- Exact Symmetries: not only the metric admits Killing vectors but also fluxes are invariant [19-21, 28, 29].

- Partially Broken Symmetries: Metric invariant, some fluxes are not

- Broken Symmetries: Metric and fluxes only asymptotically invariant [22, 23, 42, 43]

The PT ansatz [5] enjoys $\mathrm{SU}(2) \times \widetilde{\mathrm{SU}(2)}$ isometry for arbitrary choices of the 'radial' functions. On the contrary, the $\mathrm{U}(1)_{R}$, associated to shifts of the coordinate $\psi$, is broken except for very special cases. The breaking is spontaneous from the bulk viewpoint, i.e. the would-be massless vector field becomes massive after 'eating' a Goldstone boson. The Stückelberg formalism for the gauging of axionic shift symmetries is particularly convenient in this respect [19-23, 28, 29, 42, 43]. Except for some very general remarks, we will neither have much further to say about broken symmetries and massive vectors nor discuss at all massless vectors related to harmonic forms [7] and to probe branes [37]. The latter give rise to chiral 'flavor' symmetries (breaking) and mesons. The former to baryonic symmetries.

The plan of the paper is as follows. In section 2 we describe the 5-d Lagrangian governing the dynamics of gauge fields and their mixing with would-be axions. Next, in section 3, we check that the PT ansatz indeed admits full $\mathrm{SU}(2) \times \widetilde{\mathrm{SU}(2)}$ symmetry, in that not only the metric but also the other background field (strengths) are invariant. In section 4, we identify the bulk vector fields that remain massless by means of an ansatz for the fluctuations of the metric and $p$-forms, that diagonalizes the coupled equations. Finally, in section 5 we illustrate the procedure in the simple case of holographic flows and other flux vacua with $F_{3}$ only, which are invariant under $\Omega$. Specifically we study the MN solution [4] associated to wrapped D5-branes, i.e. 'fractional' D3-branes. Our conclusions and summary are contained in section 6 .

\section{Vector fields in holographic renormalization}

The 5-d Lagrangian describing vector fields and their possible mixing with inert (pseudo)scalars e.g. axions reads [19-21, 28, 29]

$$
\mathcal{L}_{v-a}=-\frac{1}{4} \mathcal{K}_{i j} F_{\mu \nu}^{i} F^{\mu \nu j}+\frac{1}{2} h_{A B}\left(\partial_{\mu} \beta^{A}-M_{i}^{A} A_{\mu}^{i}\right)\left(\partial^{\mu} \beta^{B}-M_{j}^{B} A^{\mu j}\right)
$$

The gauge kinetic function $\mathcal{K}_{i j}$ and the axion metric $h_{A B}$ may depend on the (active) scalars, while, in the present parametrization $M_{i}^{A}$ are constant mass parameters. ${ }^{2}$ The

\footnotetext{
${ }^{2}$ In general, after gauging some isometry of the scalar metric $\mathcal{G}_{a b}(\phi)$, covariant derivatives are given by $D \phi^{a}=\partial \phi^{a}+\mathcal{K}_{i}^{a}(\phi) A^{i}$, where $\mathcal{K}_{i}^{a}$ are Killing vectors i.e. $\nabla_{\mathcal{G}}^{a} \mathcal{K}_{i}^{b}(\phi)+\nabla_{\mathcal{G}}^{b} \mathcal{K}_{i}^{a}(\phi)=0, \forall i$. Here we focus on the gauging of axionic shifts $\delta \beta^{A}=M_{i}^{A} \alpha_{0}^{i}$.
} 
Lagrangian is invariant under gauge transformations of the form

$$
\delta A^{i}=\partial \alpha^{i} \quad, \quad \delta \beta^{A}=M_{i}^{A} \alpha^{i} .
$$

The square mass matrix

$$
\mathcal{M}_{i j}^{2}(\phi)=h_{A B} M_{i}^{A} M_{j}^{B}
$$

is semi-positive definite. Zero eigenvalues correspond to exactly massless vectors, associated to isometries or to harmonic forms present in the background solution. Non-zero eigenvalues correspond to massive vectors and broken symmetries. Introducing gauge invariant combinations for the latter

$$
\mathcal{B}_{\mu}^{\hat{i}}=A_{\mu}^{\hat{i}}-\left(\mathcal{M}_{\perp 0}^{-2}\right)^{\hat{i} \hat{j}} M_{\hat{j}}^{A} h_{A B} \partial_{\mu} \beta^{B}
$$

and denoting by $A_{\mu}^{i_{o}}$ the former yields

$$
\mathcal{L}_{v}=-\frac{1}{4} \mathcal{K}_{i_{o} j_{o}}(\phi) F_{\mu \nu}^{i_{o}} F^{\mu \nu j_{o}}-\frac{1}{4} \mathcal{K}_{\hat{i} \hat{j}}(\phi) \mathcal{F}_{\mu \nu}^{\hat{i}} \mathcal{F}^{\mu \nu \hat{j}}+\frac{1}{2} \mathcal{M}_{\hat{i} \hat{j}}^{2}(\phi) \mathcal{B}_{\mu}^{\hat{i}} \mathcal{B}^{\mu \hat{j}}
$$

After diagonalization, one finds a collection of decoupled vector bosons ${ }^{3}$ each described by

$$
\mathcal{L}_{v}=-\frac{1}{4} \mathcal{K}(\phi) \mathcal{F}_{\mu \nu} \mathcal{F}^{\mu \nu}+\frac{1}{2} \mathcal{M}^{2}(\phi) \mathcal{A}_{\mu} \mathcal{A}^{\mu}
$$

where $\mathcal{K}(\phi)$ is the resulting gauge kinetic function and $\mathcal{M}(\phi)$ is the possibly vanishing mass.

Putting the kinetic term in canonical form one has [19]

$$
\mathcal{M}_{\text {eff }}^{2}=\frac{1}{2} \frac{\mathcal{K}^{\prime \prime}}{\mathcal{K}}+\frac{A^{\prime} \mathcal{K}^{\prime}}{\mathcal{K}}-\frac{1}{4}\left(\frac{\mathcal{K}^{\prime}}{\mathcal{K}}\right)^{2}+\frac{\mathcal{M}^{2}}{\mathcal{K}}
$$

where primes denote derivatives wrt the holographic radial variable. Clearly $\mathcal{M}^{2}=0$ for bulk vector fields associated to unbroken boundary currents (exact global symmeries), while $\mathcal{M}^{2} \neq 0$ for broken symmetries. In most if not all known cases [19]

$$
\mathcal{M}_{\text {eff }}^{2}=-2 A^{\prime \prime}
$$

Quite remarkably but without a clear explanation, the above relation has been verified for (transverse) vectors fields in all known solutions: Coulomb branch flow with $\mathrm{SO}(6) \rightarrow$ $\mathrm{SO}(4) \times \mathrm{SO}(2)$ [19-21, 28, 29], GPPZ flow $\mathrm{SO}(6) \rightarrow \mathrm{SO}(3) \times \mathrm{U}(1)_{R}$ [19-21], $\mathrm{KT}$ (and partially KS) solution with (broken) $\mathrm{U}(1)_{R}$ R-symmetry [22-25].

\section{$3 \quad$ Field equations and PT ansatz}

To set the stage for our analysis, let us now briefly recall Papadopoulos and Tseytlin (PT) ansatz for flux vacua in Type IIB supergravity and its symmetries. The main motivation behind PT ansatz is to identify a subset of fields that form a consistent truncation of Type IIB supergravity and allow to study flux vacua with reduced or no supersymmetry at all.

\footnotetext{
${ }^{3}$ At the quadratic level this is true for non-abelian symmetries, too.
} 
The reader familiar with Type IIB supergravity and the PT ansatz can skip the following part and go directly to section 3.2 .

In the Einstein frame, Type IIB supergravity equations read

$$
\begin{aligned}
& R_{M N}=\frac{1}{2} \partial_{M} \phi \partial_{N} \phi+\frac{1}{2} e^{2 \phi} \partial_{M} \chi \partial_{N} \chi+\frac{1}{96} \hat{G}_{M P Q K L} \hat{G}_{N} P Q K L \\
& \frac{1}{4} e^{\phi} \hat{F}_{M P Q} \hat{F}_{N}{ }^{P Q}+\frac{1}{4} e^{-\phi} H_{M P Q} H_{N}{ }^{P Q}-\frac{1}{48} g_{M N}\left[e^{\phi} \hat{F}_{L P Q} \hat{F}^{L P Q}+e^{-\phi} H_{L P Q} H^{L P Q}\right] \\
& \nabla^{2} \phi=e^{2 \phi} \partial_{M} \chi \partial^{M} \chi+\frac{1}{12} e^{\phi} \hat{F}_{L M N} \hat{F}^{L M N}-\frac{1}{12} e^{-\phi} H_{L M N} H^{L M N} \\
& \nabla^{M}\left(e^{2 \phi} \partial_{M} \chi\right)=-\frac{1}{6} e^{\phi} H_{L M N} F^{L M N} \\
& \nabla^{M}\left(e^{\phi} \hat{F}_{M N P}\right)=\frac{1}{6} G_{N P Q R S} H^{Q R S} \\
& \nabla^{M}\left(e^{-\phi} H_{M N P}-e^{\phi} \chi \hat{F}_{M N P}\right)=-\frac{1}{6} G_{N P Q R S} F^{Q R S} \\
& \hat{G}_{M_{1} \ldots M_{5}}=\frac{1}{120} \varepsilon_{M_{1} \ldots M_{5} M_{6} \ldots M_{10}} \hat{G}^{M_{6} \ldots M_{10}}
\end{aligned}
$$

where

$$
\hat{F}_{3}=F_{3}-\chi H_{3} \quad \hat{F}_{5}=F_{5}+B_{2} F_{3}
$$

with

$$
F_{1}=d \chi \quad F_{3}=d C_{2} \quad F_{5}=d A_{4} \quad H_{3}=d B_{2}
$$

\subsection{PT ansatz}

The consistent truncation of 10-d Type IIB supergravity found by Papadopoulos and Tseytlin is based on the following ansatz.

- Metric

$$
\begin{aligned}
d s_{10}^{2}= & e^{2 p(u)-x(u)}\left(e^{2 A(u)} d x \cdot d x+N_{5} d u^{2}\right)+N_{5}\left[e^{x(u)+g(u)}\left(e_{1}^{2}+e_{2}^{2}\right)\right. \\
& \left.+\frac{1}{4} e^{x(u)-g(u)}\left(\tilde{\omega}_{1}^{2}+\tilde{\omega}_{2}^{2}\right)+\frac{1}{4} e^{-6 p(u)-x(u)} \tilde{\omega}_{3}^{2}\right]
\end{aligned}
$$

where $u$ denotes the holographic radial variable, the functions $A, p, x, g$ depend on $u$, and the 'invariant' one-forms read

$$
\begin{aligned}
& e_{1}=d \theta, \quad e_{2}=-\sin \theta d \varphi \\
& \tilde{\omega}_{1}=\omega_{1}-a(u) e_{1}, \quad \tilde{\omega}_{2}=\omega_{2}-a(u) e_{2}, \quad \tilde{\omega}_{3}=\omega_{3}-\cot \theta e_{2} \\
& \omega_{1}=\sin \psi \sin \tilde{\theta} d \tilde{\varphi}+\cos \psi d \tilde{\theta}, \quad \omega_{2}=-\sin \psi d \tilde{\theta}+\cos \psi \sin \tilde{\theta} d \tilde{\varphi} \\
& \omega_{3}=d \psi+\cos \tilde{\theta} d \tilde{\varphi}
\end{aligned}
$$

- NS-NS dilaton and R-R axion

$$
\phi=\phi(u) \quad, \quad \chi=0
$$


- NS-NS 3-form

$$
\begin{aligned}
H_{3}= & h_{2}(u) \tilde{\omega}_{3} \wedge\left(\omega_{1} \wedge e_{1}+\omega_{2} \wedge e_{2}\right)+d u \wedge\left[h_{1}^{\prime}(u)\left(\omega_{1} \wedge \omega_{2}+e_{1} \wedge e_{2}\right)\right. \\
& \left.+h_{2}^{\prime}(u)\left(\omega_{1} \wedge e_{2}-\omega_{2} \wedge e_{1}\right)+h_{3}^{\prime}(u)\left(-\omega_{1} \wedge \omega_{2}+e_{1} \wedge e_{2}\right)\right]
\end{aligned}
$$

where ' denotes derivative wrt $u$. Since $d H_{3}=0$, one has $H_{3}=d B_{2}$ with

$$
\begin{aligned}
B_{2}= & h_{1}(u)\left(e_{1} \wedge e_{2}+\omega_{1} \wedge \omega_{2}\right)+h_{2}(u)\left(\omega_{1} \wedge e_{2}-\omega_{2} \wedge e_{1}\right) \\
& +h_{3}(u)\left(-\omega_{1} \wedge \omega_{2}+e_{1} \wedge e_{2}\right)
\end{aligned}
$$

- R-R 3-form

$$
\begin{aligned}
F_{3}= & \frac{N_{5}}{4}\left\{\tilde{\omega}_{3} \wedge\left[\left(\omega_{1} \wedge \omega_{2}+e_{1} \wedge e_{2}\right)-b(u)\left(\omega_{1} \wedge e_{2}-\omega_{2} \wedge e_{1}\right)\right]\right. \\
& \left.+b^{\prime}(u) d u \wedge\left(\omega_{1} \wedge e_{1}+\omega_{2} \wedge e_{2}\right)\right\}
\end{aligned}
$$

since $d F_{3}=0$, one has $F_{3}=d C_{2}$ with

$$
C_{2}=\frac{N_{5}}{4}\left[\psi\left(e_{1} \wedge e_{2}+\omega_{1} \wedge \omega_{2}\right)+b(u)\left(\omega_{1} \wedge e_{1}+\omega_{2} \wedge e_{2}\right)+\cos \theta \cos \tilde{\theta} d \varphi d \tilde{\varphi}\right]
$$

- R-R self-dual 5-form

$$
\hat{G}_{5}=\mathcal{G}_{5}+* \mathcal{G}_{5} \quad \text { with } \quad \mathcal{G}_{5}=K(u) e_{1} \wedge e_{2} \wedge \omega_{1} \wedge \omega_{2} \wedge \omega_{3}
$$

where $\hat{G}_{5}=G_{5}+B_{2} \wedge F_{3}$ with $G_{5}=d C_{4}$.

Integrating $\hat{G}_{5}=d C_{4}+B_{2} \wedge F_{3}$ over a closed 'internal' 5-d section at fixed $u$ yields

$$
K(u)=N_{3}+2 N_{5}\left[h_{1}(u)+b(u) h_{2}(u)\right]
$$

that allows to eliminate $K$ in terms of $b, h_{1}, h_{2}$ and the integers $N_{3}$ and $N_{5}$ (i.e. number of D3- and D5-branes in the UV). The Bianchi identity for $H_{3}$ yields

$$
d h_{3}=\frac{\left(e^{2 g}+2 a^{2}+e^{-2 g} a^{4}-e^{-2 g}\right) d h_{1}+2 a\left(1-e^{-2 g}+a^{2} e^{-2 g}\right) d h_{2}}{e^{2 g}+\left(1-a^{2}\right)^{2} e^{-2 g}+2 a^{2}}
$$

that allows one to eliminate $h_{3}$, too.

The remaining scalar fields $\left\{p, x, g, a, b, \phi, h_{1}, h_{2}\right\}$ are governed by a 5 -d effective Lagrangian with (almost) diagonal metric $\mathcal{G}_{a b}$ (only $h_{1}$ and $h_{2}$ mix with each other) and a complicated potential that play no role in our analysis.

\subsection{Killing vectors}

For arbitrary choices of the functions $x, g, p, a, \phi, b, h_{1}, h_{2},\left(h_{3}, K\right)$ of the radial coordinate $u$, the metric and $p$-forms are invariant under $\mathrm{SU}(2) \times \widetilde{\mathrm{SU}(2)}$ isometry generated by the six 
Killing vectors $\xi_{a}$

$$
\begin{aligned}
\xi_{+} & \equiv \xi_{1}=e^{i \varphi}(0,0,0,0,0,-1,0,-i \cot \theta, 0, i \csc \theta) \\
\xi_{-} & \equiv \xi_{2}=e^{-i \varphi}(0,0,0,0,0,1,0,-i \cot \theta, 0, i \csc \theta) \\
\xi_{3} & \equiv \xi_{3}=(0,0,0,0,0,0,0,1,0,0) \\
\tilde{\xi}_{+} & \equiv \xi_{4}=e^{i \tilde{\varphi}}(0,0,0,0,0,0,-1,0,-i \cot \tilde{\theta}, i \csc \tilde{\theta}) \\
\tilde{\xi}_{-} & \equiv \xi_{5}=e^{-i \tilde{\varphi}}(0,0,0,0,0,0,1,0,-i \cot \tilde{\theta}, i \csc \tilde{\theta}) \\
\tilde{\xi}_{3} & \equiv \xi_{6}=(0,0,0,0,0,0,0,0,1,0)
\end{aligned}
$$

Notice that $\xi_{a}$ have only components in the internal directions, i.e. $\xi^{M}=\delta_{i}^{M} \xi^{i}$ with $M=1, \ldots, 10$ and $i=6, \ldots, 10$, and the contra-variant components displayed above only depend on the internal 'angular' variables, i.e. $\partial_{\mu} \xi^{M}=0$ with $\mu=1, \ldots, 5$. Although the metric does not mix the angular variables with the non-compact variables, after lowering the indices the components of the Killing vector acquire a $u$ dependence due to warping. Clearly PT preserves Poincarè symmetry in the 'boundary' space-time directions, too.

It is easy to check that also the following two-forms

$$
\begin{aligned}
e_{1} & \wedge e_{2}=-\sin \theta d \theta \wedge d \varphi=d \cos \theta \wedge d \varphi \\
\omega_{1} & \wedge \omega_{2}=+\sin \tilde{\theta} d \tilde{\theta} \wedge d \tilde{\varphi}=-d \cos \tilde{\theta} \wedge d \tilde{\varphi} \\
\omega_{1} \wedge e_{1}+\omega_{2} & \wedge e_{2}=(\sin \psi \sin \tilde{\theta} d \tilde{\varphi}+\cos \psi d \tilde{\theta}) \wedge d \theta+\sin \theta(\sin \psi d \tilde{\theta}-\cos \psi \sin \tilde{\theta} d \tilde{\varphi}) \wedge d \varphi \\
\omega_{1} \wedge e_{2}-\omega_{2} & \wedge e_{1}=-\sin \theta(\sin \psi \sin \tilde{\theta} d \tilde{\varphi}+\cos \psi d \tilde{\theta}) \wedge d \varphi+(\sin \psi d \tilde{\theta}-\cos \psi \sin \tilde{\theta} d \tilde{\varphi}) \wedge d \theta
\end{aligned}
$$

as well as the one-form

$$
\tilde{\omega}_{3}=d \psi+\cos \tilde{\theta} d \tilde{\varphi}+\cos \theta d \varphi
$$

are $\mathrm{SU}(2) \times \widetilde{\mathrm{SU}(2)}$ invariant, in the sense that $\mathcal{L}_{\xi}(\ldots)=0{ }^{4}$

As a consequence, all background field-strengths are invariant i.e.

$$
\mathcal{L}_{\xi_{a}} H_{3}=0, \quad \mathcal{L}_{\xi_{a}} F_{3}=0, \quad \mathcal{L}_{\xi_{a}} G_{5}=0
$$

Moreover, since

$$
\mathcal{L}_{\xi_{a}} B_{2}=0
$$

one also has

$$
\mathcal{L}_{\xi_{a}} \hat{F}_{3}=0, \quad \mathcal{L}_{\xi_{a}} \hat{G}_{5}=0
$$

while, in the chosen gauge,

$$
\mathcal{L}_{\xi_{a}} C_{2} \neq 0
$$

\footnotetext{
${ }^{4}$ Lie derivatives act according to

$$
\mathcal{L}_{v} T_{M_{1} \ldots M_{p}}{ }^{N_{1} \ldots N_{q}}=v^{L} \partial_{L} T_{M_{1} \ldots M_{p}}{ }^{N_{1} \ldots N_{q}}+\sum_{i} T_{M_{1} \cdot L \cdot M_{p}}{ }^{N_{1} \ldots N_{q}} \partial_{M_{i}} v^{L}-\sum_{j} T_{M_{1} \ldots M_{p}}{ }^{N_{1} \cdot L \cdot N_{q}} \partial_{L} v^{N_{j}} .
$$
}


By a change of gauge $\delta C_{2}=d \lambda_{1}^{C}$ we expect

$$
\mathcal{L}_{\xi_{a}} C_{2}^{\prime}=0
$$

Finally, though rather obviously, $\mathcal{L}_{\xi_{a}} \phi=0, \mathcal{L}_{\xi_{a}} \chi=0$.

While admitting $\mathrm{SU}(2) \times \widehat{\mathrm{SU}(2)}$ isometry, the $\mathrm{PT}$ ansatz generically 'breaks' the abelian isometry associated to the vector field $\hat{\xi}^{M} \partial_{M}=\partial / \partial_{\psi}$. The latter may be identified with the 'anomalous' U(1) R-symmetry of the dual $\mathcal{N}=1$ SYM theory on the boundary. In the bulk it is broken to $Z_{2 N}$ by the background 3-form and 5 -form and then broken to $Z_{2}$ by non-perturbative effects (string or D-brane instantons, depending on the choice of wrapped branes). As discussed in section 2, the bulk counterpart of the anomalous divergence of the R-symmetry current is a Higgs or rather Stückelberg mechanism [42], whereby a would-be massless vector field eats an axion and becomes massive. This effect has been studied in some details in $[22,23]$ in the case of the KT background (a singular 'relative' of KS solution), confirming the expected value for the 'mass' predicted by [19]. For the case of MN solution, some considerations about the required axion can be found in $[4,43]$.

\subsection{Discrete symmetries and closed subsectors}

There are two $Z_{2}$ symmetries and their product that allow to truncate Type IIB field equations in $D=10$ to closed sets of fields mixing only with one another. The first is world-sheet parity $\Omega$. The second is fermion parity in the L-moving sector $(-)^{F_{L}}$, which is S-dual to $\Omega$ i.e. $(-)^{F_{L}}=S \Omega S^{-1}$, where $\mathrm{S}$ exchanges $F_{3}$ and $H_{3}$ and sends $\tau=\chi+i e^{-\phi}$ to $-1 / \tau$. The Einstein-frame metric and the dilaton are invariant under both $\Omega$ and $(-)^{F_{L}}$, while the action on the other bosonic fields is

$$
\begin{array}{rll} 
& \Omega & (-)^{F_{L}} \\
\chi & - & - \\
B_{2} & - & + \\
C_{2} & + & - \\
A_{4} & - & -
\end{array}
$$

Later on we will focus on the subsector invariant under $\Omega$. For the PT ansatz, this means

$$
h_{1}=h_{2}=0 \rightarrow h_{3}=K=0
$$

MN solution for wrapped D5-branes [4] belongs to this class, i.e. it is invariant under $\Omega$. Its dual wrapped NS5-brane solution belongs to the class invariant under $(-)^{F_{L}}$. Standard $A d S_{5} \times S^{5}$, i.e. near-horizon D3-branes, is invariant under $(-)^{F_{L}} \Omega$. Finally KS and KT solutions (related to the conifold) do not preserve any of the above discrete symmetries and are thus more involved to study [26, 27].

\section{Exact symmetries and massless vectors}

In this section, we would like to discuss the fate of the $\mathrm{SU}(2) \times \widetilde{\mathrm{SU}(2)}$ isometry, that should correspond to the global 'flavor' symmetry of the boundary theory, possibly acting 
trivially on the lowest states relevant in the deep IR. Even if from the vantage point of the holographic duality, the presence of this isometry might be annoying, the analysis is quite general and applies to any isometry in any flux compactification.

We will find that the Killing vectors generating $\widetilde{\mathrm{SU}(2)}$ are associated to truly massless vectors in the bulk that correspond to an exact global 'flavor' symmetry of any solution based on PT ansatz. For the first $\mathrm{SU}(2)$ factor the situation is subtler, at least in the case of MN solution [4].

First of all notice that invariance of the metric under isometry generated by a Killing vector $\xi^{M}$ reads

$$
\mathcal{L}_{\xi} g_{M N} \equiv \nabla_{M} \xi_{N}+\nabla_{N} \xi_{M}=0
$$

that implies

$$
\nabla_{M} \xi^{M}=0
$$

as well as

$$
\nabla_{M} \xi_{N}=\frac{1}{2}\left(\nabla_{M} \xi_{N}-\nabla_{N} \xi_{M}\right)
$$

and

$$
\nabla_{L} \nabla_{M} \xi_{N}=-R_{M N L K} \xi^{K}
$$

Invariance under diffeomorphisms suggests the existence of a trivial massless zero-mode for the metric fluctuations

$$
\delta_{\text {diff }} g_{M N}=\nabla_{M} \beta_{N}+\nabla_{N} \beta_{M}
$$

Taking $\beta_{M}=\alpha(x) \xi_{M}$

$$
\delta_{\text {diff }} g_{M N}=\xi_{N} \nabla_{M} \alpha+\xi_{M} \nabla_{N} \alpha
$$

It suggests an ansatz for the metric fluctuations of the form

$$
\delta_{\text {phys }} g_{M N}=-\xi_{N} A_{M}-\xi_{M} A_{N}
$$

with $\xi^{M} A_{M}=0$ (i.e. $\delta g^{L}{ }_{L}=0$ ) and $\mathcal{L}_{\xi} A_{M}=0$, since $A_{M}=A_{M}(x)$ is to be independent of the five internal coordinates the Killing vectors act on. Gauge invariance under $\delta A_{M}^{(0)}=$ $-\nabla_{M} \alpha$ would then result from general covariance and should imply massless-ness of the vector field $A_{M}$. However, due to the presence of fluxes in the background, the story is not so simple. The metric fluctuations mix with $p$-form fluctuations, which we turn now our attention onto.

Let us then consider the general case of an $n$-form $X_{n}$, whose background $(n+1)$-form field strength $Y_{n+1}=d X_{n}$ is invariant under some isometry generated by a Killing vector $\xi$

$$
\mathcal{L}_{\xi} Y_{n+1}=i_{\xi} d Y_{n+1}+d\left(i_{\xi} Y_{n+1}\right)=0
$$

Thanks to Bianchi identity $d Y_{n+1}=0$ one has (locally)

$$
i_{\xi} Y_{n+1}=d Z_{n-1}^{\xi}
$$

where $Z_{n-1}^{\xi}$ is a $(n-1)$-form defined up to an exact form $\delta Z_{n-1}^{\xi}=d W_{n-2}$. 
Under a diffeomorphism generated by $v^{M}=\alpha \xi^{M}$

$$
\delta_{\text {Diff }} X_{n}=\alpha i_{\xi} Y_{n+1}+d\left(\alpha i_{\xi} X_{n}\right)=\alpha d Z_{n-1}^{\xi}+d\left(\alpha i_{\xi} X_{n}\right)=d \alpha \wedge Z_{n-1}^{\xi}+d\left[\alpha\left(i_{\xi} X_{n}-Z_{n-1}^{\xi}\right)\right]
$$

The last term can be cancelled by a gauge transformation of the $n$-form $X_{n}$. This suggest that the correct ansatz for the 'massless' vector $A_{1}$ associated to the coupled fluctuations of the metric and $n$-form $X_{n}$ along $\xi$ be of the form

$$
\delta X_{n}=A_{1} \wedge Z_{n-1}^{\xi}
$$

In this way gauge invariance under $\delta A_{1}=d \alpha$ would not only be a consequence of general covariance but also of the $n$-form gauge invariance. For the fluctuations of the $(n+1)$-form field-strength $Y_{n+1}$ one then finds

$$
\delta Y_{n+1}=d A \wedge Z_{n-1}^{\xi}-A \wedge d Z_{n-1}^{\xi}=d A \wedge Z_{n-1}^{\xi}-A_{1} \wedge i_{\xi} Y_{n+1}
$$

In principle the procedure applies to any background $n$-form in PT or even more general flux vacua. The analysis can be performed in quite general terms but it drastically simplifies in backgrounds where $F_{5}=0, H_{3}=0, F_{1}=0$, thanks to invariance under worldsheet parity $\Omega$, or else where $F_{5}=0, F_{3}=0, F_{1}=0$, thanks to invariance under $(-)^{F_{L}}$. In both cases mixing between $C_{2}$ and $B_{2}$ are excluded, and one can safely set $A_{4}=0$ and even $\delta \phi=0$, as we will see.

Henceforth we will focus on the sub-sector invariant under $\Omega$ i.e. $C_{2}, g, \phi$ and set $B_{2}, A_{4}, \chi$ to zero both in the background and in the fluctuations.

\section{1 $\Omega$ invariant massless vectors (i.e. $F_{5}=0$ and $H_{3}=0$ )}

Taking into account that $\mathcal{L}_{\xi} F_{3}=d\left(i_{\xi} F_{3}\right)=0$, for any exact Killing vector, one can locally write

$$
i_{\xi} F_{3}=d \mu_{1}^{\xi}
$$

that suggests the following combined ansatze for the physical fluctuations

$$
\delta g^{M N}=A^{M} \xi^{N}+A^{N} \xi^{M}, \quad \delta C_{M N}=A_{M} \mu_{N}^{\xi}-A_{N} \mu_{M}^{\xi}
$$

or, equivalently, for the latter $\delta C_{2}=A_{1} \wedge \mu_{N}^{\xi}$ so that

$$
\delta F_{3}=d \delta C_{2}=d A \wedge \mu_{1}^{\xi}-A \wedge i_{\xi} F_{3}
$$

Setting $\delta B_{2}=0, \delta \chi=0, \delta A_{4}=0$ as well as $\delta \phi=0, \delta g_{\mu \nu}=0, \delta g_{i j}=0$ one has $g^{M N} \delta g_{M N}=0$, i.e. $\delta \sqrt{\|g\|}=0$.

Moreover, $\delta g_{M N}=-A_{M} \xi_{N}-A_{N} \xi_{M}$ so that

$$
\delta g_{\mu i}=-A_{\mu} \xi_{i}=\delta g_{i \mu}
$$

while

$$
\delta C_{\mu i}=A_{\mu} \mu_{i}^{\xi}=-\delta C_{i \mu}
$$


and

$$
\begin{aligned}
\delta F_{i j k} & =0 \\
\delta F_{\mu \nu \rho} & =0 \\
\delta F_{\mu i j} & =-A_{\mu}\left(\partial_{i} \mu_{j}^{\xi}-\partial_{j} \mu_{i}^{\xi}\right) \\
\delta F_{\mu \nu i} & =\left(\partial_{\mu} A_{\nu}-\partial_{\nu} A_{\mu}\right) \mu_{i}^{\xi}-\left(A_{\mu} \partial_{\nu} \mu_{i}^{\xi}-A_{\nu} \partial_{\mu} \mu_{i}^{\xi}\right)
\end{aligned}
$$

\subsubsection{Dilaton equation (consistency check)}

Let us first check that it be consistent to set $\delta \phi=0$. Using the 3-form ansatz, one finds

$$
\begin{aligned}
\delta F^{2} & =\delta F_{L M N} F^{L M N}+3 F_{L M N} \delta g^{L P} F_{P}^{M N} \\
& =F^{i j k} \delta F_{i j k}+3 F^{u j k} \delta F_{u j k}+6 F_{u j k} \delta g^{u i} F_{i}^{j k} \\
& =-3 A_{u} F^{u j k}\left(\partial_{j} \mu_{k}^{\xi}-\partial_{k} \mu_{j}^{\xi}\right)+6 \delta g^{u i} F_{u j k} F_{i}{ }^{j k}
\end{aligned}
$$

For a solution of the PT kind one has $F_{u j k} F_{i}{ }^{j k}=0, i=6, \ldots, 10$. Also one obtains $F^{u j k}\left(\partial_{j} \mu_{k}^{\xi}-\partial_{k} \mu_{j}^{\xi}\right)=0$ for each one of the six Killing vectors $\xi_{a}$. Therefore $\delta F^{2}=0$, consistently with the ansatz $\delta \phi=0$.

\subsubsection{3-form equation}

Let us focus on the 3 -form equation

$$
\begin{aligned}
\delta\left(\nabla_{M}\left(\sqrt{g} e^{\phi} F^{M N P}\right)\right)= & \frac{1}{\sqrt{g}} \partial_{M}\left(\sqrt { g } e ^ { \phi } \left[\delta g^{M L} F_{L}{ }^{N P}+\delta g^{N L} F_{L}^{M}\right.\right. \\
& \left.\left.+\delta g^{P L} F^{M N}{ }_{L}+g^{M L} g^{N K} g^{P Q} \delta F_{L K Q}\right]\right)
\end{aligned}
$$

Decomposing into space-time $(\nu=1, \ldots, 5)$ and internal indices $(j=6, \ldots, 10)$ one has

- Equations $N=\nu, P=\rho$

$$
\begin{aligned}
\delta\left(\nabla_{M}\left(\sqrt{g} e^{\phi} F^{M \nu \rho}\right)\right) \equiv & \frac{1}{\sqrt{g}} \partial_{M}\left(\sqrt { g } e ^ { \phi } \left[\delta g^{M L} F_{L}{ }^{\nu \rho}+\delta g^{\nu L} F^{M}{ }_{L}^{\rho}\right.\right. \\
& \left.\left.+\delta g^{\rho L} F^{M \nu}{ }_{L}+g^{M L} g^{\nu K} g^{\rho Q} \delta F_{L K Q}\right]\right)=0
\end{aligned}
$$

Keeping only non-vanishing components yields

$$
\frac{1}{\sqrt{g}} \partial_{M}\left(\sqrt{g} e^{\phi}\left[\delta g^{\nu l} F^{M}{ }^{\rho}+\delta g^{\rho L} F_{L}^{M \nu}+g^{M L} g^{\nu k} g^{\rho \mu} \delta F_{L k \mu}\right]\right)=0
$$

Plugging the anstaze one eventually finds

$$
e^{\phi} \partial_{i}\left\{\sqrt{\hat{g}}\left[A^{\nu} \xi^{l} F_{l}^{i}{ }_{l}^{u} \delta_{u}^{\rho}+A^{\rho} \xi^{l} \delta_{u}^{\nu} F_{l}^{i u}+g^{i l} g^{\nu \lambda} g^{\rho \sigma}\left(\mu_{l}^{\xi} f_{\lambda \sigma}-A_{\lambda} \partial_{\sigma} \mu_{l}^{\xi}+A_{\sigma} \partial_{\lambda} \mu_{l}^{\xi}\right)\right]\right\}=0
$$


where $f_{M N}=\partial_{M} A_{N}-\partial_{N} A_{M}$ and $\sqrt{\hat{g}}$ denote the dependence of $\sqrt{g}$ on the internal coordinates. Finally, using $i_{\xi} F_{3}=d \mu_{\xi}$ one arrives at the following constraint for $\mu_{\xi}$

$$
0=\partial_{i}\left(\sqrt{\hat{g}} \mu^{i}\right) f^{\nu \rho} \Rightarrow \nabla_{M} \mu^{M}=0
$$

One can check that this is satisfied for all $\mu_{\xi}$ in any background of the PT kind. Anyway, we expect that it should always be possible to satisfy the constraint by adding to $\mu^{\xi}$ an exact form $d \eta^{\xi}$, where $\eta^{\xi}$ is an appropriate function.

- Equations for $N=i, P=j$

$$
\begin{aligned}
\delta\left(\nabla_{M}\left(\sqrt{g} e^{\phi} F^{M i j}\right)\right)= & \frac{1}{\sqrt{g}} \partial_{M}\left(\sqrt { g } e ^ { \phi } \left[\delta g^{M L} F_{L}{ }^{i j}+\delta g^{i L} F^{M}{ }_{L}^{j}\right.\right. \\
& \left.\left.+\delta g^{j L} F^{M i}{ }_{L}+g^{M L} g^{i K} g^{j Q} \delta F_{L K Q}\right]\right)=0
\end{aligned}
$$

Keeping only non-vanishing components yields

$$
\frac{1}{\sqrt{g}} \partial_{M}\left(\sqrt{g} e^{\phi}\left[\delta g^{M L} F_{L}{ }^{i j}+\delta g^{i L} F^{M} L^{j}-\delta g^{j L} F_{L}^{M}{ }^{i}+g^{M L} g^{i k} g^{j l} \delta F_{L k l}\right]\right)=0
$$

Plugging the anstaze one eventually finds

$$
\begin{aligned}
& \frac{e^{\phi}}{\sqrt{\hat{g}}} A^{u} \partial_{m}\left\{\sqrt{\hat{g}}\left[\xi^{m} F_{u}^{i j}+\xi^{i} F_{u}^{m j}-\xi^{j} F_{u}^{m i}\right]\right\} \\
& \quad+\frac{1}{\sqrt{g}} \partial_{\mu}\left\{e^{\phi} \sqrt{g}\left[A^{\mu} \xi^{k} F_{k}^{i j}-A^{\mu}\left(\nabla^{i} \mu_{\xi}^{j}-\nabla^{i} \mu_{\xi}^{j}\right)\right]\right\}=0
\end{aligned}
$$

that is satisfied after using $\mathcal{L}_{\xi} F_{3}=0$ i.e. $i_{\xi} F_{3}=d \mu_{\xi}, \nabla_{M} \xi^{M}=0$ and the background 3-form equation $\partial_{m}\left(\sqrt{\hat{g}} F_{u}{ }^{m j}\right)=0$.

- Equations for $N=\nu, P=l$

$$
\begin{aligned}
\delta\left(\nabla_{M}\left(\sqrt{g} e^{\phi} F^{M \nu l}\right)\right)= & \frac{1}{\sqrt{g}} \partial_{M}\left(\sqrt { g } e ^ { \phi } \left[\delta g^{M L} F_{L}{ }^{\nu l}+\delta g^{\nu L} F_{L}^{M}{ }_{L}^{l}\right.\right. \\
& \left.\left.+\delta g^{l L} F_{L}^{M \nu}+g^{M L} g^{\nu K} g^{l Q} \delta F_{L K Q}\right]\right)=0
\end{aligned}
$$

Keeping only non-zero components yields

$$
\begin{aligned}
0= & \frac{1}{\sqrt{g}} \partial_{\mu}\left(\sqrt{g} e^{\phi}\left[\delta g^{\mu k} F_{k}{ }^{\nu l}+\delta g^{\nu k} F_{k}^{\mu}{ }^{l}+g^{\mu \rho} g^{\nu \lambda} g^{l j} \delta F_{\rho \lambda j}\right]\right) \\
& +e^{\phi} \partial_{i}\left(\delta g^{\nu k} F_{k}^{i}{ }^{l}+g^{i k} g^{\nu \mu} g^{l j} \delta F_{k \mu j}\right) .
\end{aligned}
$$

Plugging in the ansatz for the fluctuations yields

$$
\begin{gathered}
\frac{1}{\sqrt{g}} \partial_{\mu}\left(\sqrt { g } e ^ { \phi } \left[A^{\mu} \xi^{k} F_{k}{ }^{\nu l}+A^{\nu} \xi^{k} F_{k}^{\mu}{ }^{l}+g^{\mu \rho} g^{\nu \lambda} g^{l j}\left(f_{\rho \lambda} \mu_{j}-A_{\rho} \xi^{n} F_{n \lambda j}\right.\right.\right. \\
\left.\left.\left.+A_{\lambda} \xi^{n} F_{n \rho j}\right)\right]\right)+e^{\phi} \partial_{i}\left(A^{\nu} \xi^{k} F^{i}{ }_{k}{ }^{l}+g^{i k} g^{\nu \mu} g^{l j}\left(-A_{\mu} \xi^{n} F_{n j k}\right)\right)=0
\end{gathered}
$$

After various cancellations, one finally arrives at the Dynamical Equation for the vector fields

$$
\partial_{\mu}\left(\sqrt{g} e^{\phi} g^{\mu \rho} g^{\nu \lambda} f_{\rho \lambda} \mu_{\xi}^{l}\right)=0
$$


When $\mu_{\xi}^{i}=e^{-\phi / 2} \xi^{i}$, this further simplifies into

$$
\xi^{i} \partial_{\mu}\left(\sqrt{g} e^{\phi / 2} g^{\mu \rho} g^{\nu \lambda} f_{\rho \lambda}\right)=0
$$

which neatly displays the correspondence between bulk 5-d massless vector fields and exact Killing vectors.

\subsubsection{Einstein equations}

It is straightforward but very laborious to show that Einstein equations lead to the same results, i.e. the very same dynamical equation for $A_{\mu}$. For simplicity we will restrict our attention on the case in which $\mu_{M}^{\xi}=e^{-\phi / 2} \xi_{M}$ i.e. $i_{\xi} F_{3}=d\left(e^{-\frac{\phi}{2}} \lambda^{\xi}\right)$ where $\lambda^{\xi}=g_{M N} \xi^{M} d x^{N}$

Let us start with the source term

$$
\begin{aligned}
\delta S_{M N}= & \frac{1}{4} e^{\phi}\left(\delta F_{M P Q} F_{N} P Q+\delta F_{N P Q} F_{M}^{P Q}+2 \delta g^{P L} F_{M P Q} F_{N L}{ }^{Q}\right)-\frac{1}{48} e^{\phi} \delta g_{M N} F^{2} \\
= & \frac{1}{4}\left[e^{\frac{\phi}{2}} F_{N} P Q\right. \\
& +(M \leftrightarrow N)]-\frac{1}{48} e^{\phi} \delta g_{M N} F^{2}
\end{aligned}
$$

Defining

$$
h_{L M}=\nabla_{L} \xi_{M}-\nabla_{M} \xi_{L}=\frac{1}{2}\left(\partial_{L} \phi \xi_{M}-\partial_{M} \phi \xi_{L}\right)+e^{\phi}\left(e^{-\frac{\phi}{2}} \xi^{P} F_{P L M}\right)
$$

the first order fluctuation of the Ricci tensor reads

$$
\begin{aligned}
\delta R_{M N}= & -\frac{1}{2}\left(\nabla^{2} \delta g_{M N}-\nabla^{L} \nabla_{M} \delta g_{N L}-\nabla^{L} \nabla_{N} \delta g_{M L}+\nabla_{M} \nabla_{N} \delta g_{L}^{L}\right) \\
= & -\frac{1}{2} \nabla^{L}\left[\xi_{N} f_{M L}+\xi_{M} f_{N L}+\xi_{L}\left(\nabla_{M} A_{N}+\nabla_{N} A_{M}\right)+A_{M} h_{N L}+A_{N} h_{M L}\right] \\
= & \frac{1}{2} e^{\frac{\phi}{2}}\left[f_{L M} \nabla^{L}\left(e^{-\frac{\phi}{2}} \xi_{N}\right)+f_{L N} \nabla^{L}\left(e^{-\frac{\phi}{2}} \xi_{M}\right)\right]+\frac{1}{2} e^{-\frac{\phi}{2}}\left[\xi_{N} \nabla^{L}\left(e^{\frac{\phi}{2}} f_{L M}\right)\right. \\
& \left.+\xi_{M} \nabla^{L}\left(e^{\frac{\phi}{2}} f_{L N}\right)\right]-\frac{1}{2} \xi^{L} \nabla_{L}\left(\nabla_{M} A_{N}+\nabla_{N} A_{M}\right)+\frac{1}{2} \nabla^{L}\left(A_{M} h_{L N}+A_{N} h_{L M}\right)
\end{aligned}
$$

Moreover one finds

$$
\begin{aligned}
\frac{1}{2} \nabla^{L}\left(A_{M} h_{L N}+A_{N} h_{L M}\right)= & \frac{1}{2} \nabla^{L}\left(A_{M} e^{\frac{\phi}{2}} \xi^{P} F_{P L N}+A_{N} e^{\frac{\phi}{2}} \xi^{P} F_{P L M}\right) \\
& +\frac{1}{4} \nabla^{L}\left(A_{M} \xi_{N} \partial_{L} \phi-A_{M} \xi_{L} \partial_{N} \phi+A_{N} \xi_{M} \partial_{L} \phi-A_{N} \xi_{L} \partial_{M} \phi\right) \\
= & \frac{1}{2} e^{\frac{\phi}{2}} \xi^{P} F_{P L N} \nabla^{L} A_{M}-\frac{1}{4} e^{\phi} \xi^{k} F_{k}{ }^{L P} F_{L P N} A_{M}-\frac{1}{48} e^{\phi} \delta g_{M N} F^{2} \\
& +\frac{1}{4} \partial_{L} \phi \nabla^{L}\left(A_{M} \xi_{N}+A_{N} \xi_{M}\right)-\frac{1}{4} \xi^{L} \nabla_{L}\left(A_{M} \partial_{N} \phi+A_{N} \partial_{M} \phi\right)
\end{aligned}
$$

and also

$$
-\frac{1}{4} e^{\phi} \xi^{k} F_{k}^{L P} F_{L P N} A_{M}=-\frac{1}{4} e^{\phi} F_{N}^{P Q} \xi^{L} A_{[M} F_{L P Q]}+\frac{1}{4} e^{\phi}\left(A^{P} \xi^{L}+A^{L} \xi^{P}\right) F_{M P Q} F_{N L}{ }^{Q}
$$


Simplifying terms in the left and right hand side yields

$$
\delta^{\prime} S_{M N}=\frac{1}{4} e^{\frac{\phi}{2}} F_{N}^{P Q} f_{[M P} \xi_{Q]}+(M \leftrightarrow N)
$$

and

$$
\begin{aligned}
\delta^{\prime} R_{M N}= & \frac{1}{2} e^{\frac{\phi}{2}} f_{L M} \nabla^{L}\left(e^{-\frac{\phi}{2}} \xi_{N}\right)+\frac{1}{2} e^{-\frac{\phi}{2}} \xi_{N} \nabla^{L}\left(e^{\frac{\phi}{2}} f_{L M}\right)-\frac{1}{2} \xi^{L} \nabla_{L} \nabla_{M} A_{N} \\
& +\frac{1}{2} e^{\frac{\phi}{2}} \xi^{P} F_{P L N} \nabla^{L} A_{M}+\frac{1}{4} \partial_{L} \phi \nabla^{L}\left(A_{M} \xi_{N}\right)-\frac{1}{4} \xi^{L} \nabla_{L}\left(A_{M} \partial_{N} \phi\right)+(M \leftrightarrow N)
\end{aligned}
$$

In particular for $M=\mu$ and $N=i$, the remaining terms in the source fluctuations are

$$
\delta S_{\mu i}=\frac{1}{2} e^{-\frac{\phi}{2}}\left(\frac{1}{2} \phi^{\prime} \xi_{i}-\xi_{i}^{\prime}\right)\left(\nabla_{\mu} A_{u}-\nabla_{u} A_{\mu}\right)
$$

while the remaining terms in the Ricci fluctuations read

$$
\begin{aligned}
\frac{1}{2} e^{\frac{\phi}{2}} f_{L M} \nabla^{L}\left(e^{-\frac{\phi}{2}} \xi_{N}\right)+(M \leftrightarrow N) & =\frac{1}{4} e^{-\frac{\phi}{2}}\left(\nabla_{u} A_{\mu}-\nabla_{\mu} A_{u}\right)\left(\xi_{i}^{\prime}-\phi^{\prime} \xi_{i}\right) \\
-\frac{1}{2} \xi^{L} \nabla_{L} \nabla_{M} A_{N}+(M \leftrightarrow N) & =-\frac{1}{4} e^{-\frac{\phi}{2}} \xi_{i}^{\prime}\left(\nabla_{\mu} A_{u}+\nabla_{u} A_{\mu}\right)+\frac{1}{4} e^{-\frac{\phi}{2}} \delta_{\mu}^{u} \xi_{j}^{\prime} g^{j k} A_{u} \partial_{u} g_{k i} \\
\frac{1}{2} e^{\frac{\phi}{2}} \xi^{P} F_{P L N} \nabla^{L} A_{M}+(M \leftrightarrow N) & = \\
e^{-\frac{\phi}{2}}\left\{-\frac{1}{4} \phi^{\prime} \xi_{i} \nabla_{u} A_{\mu}\right. & \left.+\frac{1}{2} \xi_{i}^{\prime} \nabla_{u} A_{\mu}-\delta_{\mu}^{u}\left(-\frac{1}{8} \phi^{\prime} A_{u} \xi_{i}^{\prime}+\frac{1}{4} \xi_{j}^{\prime} g^{j k} A_{u} \partial_{u} g_{k i}\right)\right\} \\
\frac{1}{4} \partial_{L} \phi \nabla^{L}\left(A_{M} \xi_{N}\right)+(M \leftrightarrow N) & =e^{-\frac{\phi}{2}}\left(\frac{1}{4} \phi^{\prime} \xi_{i} \nabla_{u} A_{\mu}+\frac{1}{8} \xi_{i}^{\prime} \phi^{\prime} A_{\mu}\right)
\end{aligned}
$$

and finally

$$
-\frac{1}{4} \xi^{L} \nabla_{L}\left(A_{M} \partial_{N} \phi\right)+(M \leftrightarrow N)=e^{-\frac{\phi}{2}}\left(-\frac{1}{8} \xi_{i}^{\prime} A_{\mu} \phi^{\prime}-\frac{1}{8} \xi_{i}^{\prime} A_{u} \phi^{\prime} \delta_{\mu}^{u}\right)
$$

After a number of cancellation one eventually finds

$$
\delta S_{\mu i}=\delta R_{\mu i}
$$

when the 3-form equations are satisfied, showing that the fluctuations ansatz then satisfies Einstein equations, too.

It is easy to check that the other components $(i, j)$ and $(\mu, \nu)$ are satisfied as well. For brevity, we refrain to present the details here.

\subsection{One-forms associated to Killing vectors}

For completeness and later use, let us display in the following the expressions of the one-forms associated to the Killing vectors in a generic PT background. Setting ${ }^{5} \lambda_{\xi}=$

\footnotetext{
${ }^{5}$ The constant factor $N_{5}^{-1}$ is inserted for later notational convenience.
} 
$N_{5}^{-1} g_{i j} \xi^{i} d x^{j}$, one has

$$
\begin{aligned}
& \lambda_{1}=-\frac{1}{4} e^{i \varphi-g+x}\left(a^{2}+4 e^{2 g}\right) d \theta+\frac{1}{4} e^{i \varphi-g+x} a(\cos \psi+i \cos \theta \sin \psi) d \tilde{\theta} \\
& -\frac{1}{4} i e^{i \varphi-g-6 p-x}\left(e^{6 p+2 x} a^{2}-e^{g}+4 e^{2(g+3 p+x)}\right) \cos \theta \sin \theta d \varphi \\
& +\frac{1}{4} e^{i \varphi-g-6 p-x}\left[i e^{g} \cos \tilde{\theta} \sin \theta+e^{6 p+2 x} a(\sin \psi-i \cos \psi \cos \theta) \sin \tilde{\theta}\right] d \tilde{\varphi} \\
& +\frac{1}{4} i e^{i \varphi-6 p-x} \sin \theta d \psi \\
& \lambda_{2}=\frac{1}{4} e^{-i \varphi-g+x}\left(a^{2}+4 e^{2 g}\right) d \theta-\frac{1}{4} e^{-i \varphi-g+x} a(\cos \psi-i \cos \theta \sin \psi) d \tilde{\theta} \\
& -\frac{1}{4} i e^{-i \varphi-g-6 p-x}\left[e^{6 p+2 x} a^{2}-e^{g}+4 e^{2(g+3 p+x)}\right] \cos \theta \sin \theta d \varphi \\
& +\frac{1}{4} i e^{-i \varphi-g-6 p-x}\left[e^{g} \cos \tilde{\theta} \sin \theta-e^{6 p+2 x} a(\cos \psi \cos \theta-i \sin \psi) \sin \tilde{\theta}\right] d \tilde{\varphi} \\
& +\frac{1}{4} i e^{-i \varphi-6 p-x} \sin \theta d \psi \\
& \lambda_{3}=-\frac{1}{4} e^{x-g} a \sin \psi \sin \theta d \tilde{\theta}+\frac{1}{4}\left[e^{-6 p-x} \cos ^{2} \theta+e^{x-g}\left(a^{2}+4 e^{2 g}\right) \sin ^{2} \theta\right] d \varphi \\
& +\frac{1}{4}\left(e^{-6 p-x} \cos \theta \cos \tilde{\theta}+e^{x-g} a \cos \psi \sin \theta \sin \tilde{\theta}\right) d \tilde{\varphi}+\frac{1}{4} e^{-6 p-x} \cos \theta d \psi \\
& \lambda_{4}=\frac{1}{4} e^{i \tilde{\varphi}-g+x} a(\cos \psi+i \cos \tilde{\theta} \sin \psi) d \theta-\frac{1}{4} e^{i \tilde{\varphi}-g+x} d \tilde{\theta} \\
& +\frac{1}{4} e^{i \tilde{\varphi}-g-6 p-x}\left[e^{6 p+2 x} a(\sin \psi-i \cos \psi \cos \tilde{\theta}) \sin \theta+i e^{g} \cos \theta \sin \tilde{\theta}\right] d \varphi \\
& -\frac{1}{8} i e^{i \tilde{\varphi}-g-6 p-x}\left(-e^{g}+e^{6 p+2 x}\right) \sin (2 \tilde{\varphi}) d \tilde{\varphi}+\frac{1}{4} i e^{i \tilde{\varphi}-6 p-x} \sin \tilde{\theta} d \psi \\
& \lambda_{5}=-\frac{1}{4} e^{-i \tilde{\varphi}-g+x} a(\cos \psi-i \cos \tilde{\theta} \sin \psi) d \theta+\frac{1}{4} e^{-i \tilde{\varphi}-g+x} d \tilde{\theta} \\
& -\frac{1}{4} i e^{-i \tilde{\varphi}-g-6 p-x}\left[\left(e^{6 p+2 x} a(\cos \psi \cos \tilde{\theta}-i \sin \psi) \sin \theta-e^{g} \cos \theta \sin \tilde{\theta}\right] d \varphi\right. \\
& +\frac{1}{4} i e^{-i \tilde{\varphi}-g-6 p-x}\left(e^{g}-e^{6 p+2 x}\right) \cos \tilde{\theta} \sin \tilde{\theta} d \tilde{\varphi}+\frac{1}{4} i e^{-i \tilde{\varphi}-6 p-x} \sin \tilde{\theta} d \psi \\
& \lambda_{6}=-\frac{1}{4} e^{x-g} a \sin \psi \sin \tilde{\theta} d \theta+\frac{1}{4}\left[e^{-6 p-x} \cos \theta \cos \tilde{\theta}+e^{x-g} a \cos \psi \sin \theta \sin \tilde{\theta}\right] d \varphi \\
& +\frac{1}{4}\left[e^{-6 p-x} \cos ^{2} \tilde{\theta}+e^{x-g} \sin ^{2} \tilde{\theta}\right] d \tilde{\varphi}+\frac{1}{4} e^{-6 p-x} \cos \tilde{\theta} d \psi
\end{aligned}
$$

Then using $N_{5}^{-1} i_{\xi} F_{3}=d \mu^{\xi}$ one finds

$$
\begin{aligned}
\mu_{1}= & \frac{1}{4}\left[e^{i \varphi} f-e^{i \varphi}(f-1)\right] d \theta-\frac{1}{4} e^{i \varphi} b(\cos \psi+i \cos \theta \sin \psi) d \tilde{\theta} \\
& +\frac{1}{8}\left[i e^{i \varphi}(f-1) \sin (2 \theta)-2 i e^{i \varphi} \cos \theta(f-1) \sin \theta\right] d \varphi \\
& -\frac{1}{4} e^{i \varphi}[i \cos \tilde{\theta} \sin \theta+b(\sin \psi-i \cos \psi \cos \theta) \sin \tilde{\theta}] d \tilde{\varphi} \\
& -\frac{1}{4} i e^{i \varphi} \sin \theta d \psi
\end{aligned}
$$




$$
\begin{aligned}
\mu_{2}= & \frac{1}{4}\left[e^{-i \varphi}(f-1)-e^{-i \varphi} f\right] d \theta+\frac{1}{4} e^{-i \varphi} b(\cos \psi-i \cos \theta \sin \psi) d \tilde{\theta} \\
& +\frac{1}{8}\left[i e^{-i \varphi}(f-1) \sin (2 \theta)-2 i e^{-i \varphi} \cos \theta(f-1) \sin \theta\right] d \varphi \\
& -\frac{1}{4} i e^{-i \varphi}[\cos \tilde{\theta} \sin \theta+b(i \sin \psi-\cos \psi \cos \theta) \sin \tilde{\theta}] d \tilde{\varphi} \\
& -\frac{1}{4} i e^{-i \varphi} \sin \theta d \psi \\
\mu_{3}= & \frac{1}{4} b \sin \psi \sin \theta d \tilde{\theta}+\frac{1}{4}\left[(f-1) \sin ^{2} \theta+\left(-\cos ^{2} \theta-f \sin ^{2} \theta\right)\right] d \varphi \\
& +\frac{1}{4}(-\cos \theta \cos \tilde{\theta}-b \cos \psi \sin \theta \sin \tilde{\theta}) d \tilde{\varphi}-\frac{\cos \theta}{4} d \psi \\
\mu_{4}= & \frac{1}{4} e^{i \tilde{\varphi}} b(\cos \psi+i \cos \tilde{\theta} \sin \psi) d \theta-\frac{e^{i \tilde{\varphi}}}{4} d \tilde{\theta} \\
& \frac{1}{4} e^{i \tilde{\varphi}}[b(\sin \psi-i \cos \psi \cos \tilde{\theta}) \sin \theta+i \cos \theta \sin \tilde{\theta}] d \varphi+\frac{1}{4} i e^{i \tilde{\varphi}} \sin \tilde{\theta} d \psi \\
\mu_{5}= & -\frac{1}{4} e^{-i \tilde{\varphi}} b(\cos \psi-i \cos \tilde{\theta} \sin \psi) d \theta+\frac{e^{-i \tilde{\varphi}}}{4} d \tilde{\theta} \\
& -\frac{1}{4} i e^{-i \tilde{\varphi}}[b(\cos \psi \cos \tilde{\theta}-i \sin \psi) \sin \theta-\cos \theta \sin \tilde{\theta}] d \varphi+\frac{1}{4} i e^{-i \tilde{\varphi}} \sin \tilde{\theta} d \psi \\
\mu_{6}= & -\frac{1}{4} b \sin \psi \sin \tilde{\theta} d \theta+\frac{1}{4}(\cos \theta \cos \tilde{\theta}+b \cos \psi \sin \theta \sin \tilde{\theta}) d \varphi \\
& +\frac{1}{4} d \tilde{\varphi}+\frac{1}{4} \cos \tilde{\theta} d \psi
\end{aligned}
$$

which are valid in any PT background. We also defined $f=4 e^{2 g}+a^{2}$.

For MN background [4] a compact form for $\mu^{\xi}$ in terms of the (rescaled) KV obtains

$$
\begin{aligned}
& \mu_{(1) i}=-e^{-\frac{\phi}{2}} \xi_{(1) i}-\frac{1}{4}(f-1) e^{i \varphi}\left(\delta_{i}^{6}+i \cos \theta \sin \theta \delta_{i}^{8}\right) \\
& \mu_{(2) i}=-e^{-\frac{\phi}{2}} \xi_{(2) i}+\frac{1}{4}(f-1) e^{-i \varphi}\left(\delta_{i}^{6}-i \cos \theta \sin \theta \delta_{i}^{8}\right) \\
& \mu_{(3) i}=-e^{-\frac{\phi}{2}} \xi_{(3) i}+\frac{1}{4}(f-1) \sin ^{2} \theta \delta_{i}^{8} \\
& \mu_{(4) i}=e^{-\frac{\phi}{2}} \xi_{(4) i} \\
& \mu_{(5) i}=e^{-\frac{\phi}{2}} \xi_{(5) i} \\
& \mu_{(6) i}=e^{-\frac{\phi}{2}} \xi_{(6) i}
\end{aligned}
$$

\subsection{Scalar products and gauge kinetic functions}

The scalar products of the Killing Vectors are diagonal as a result of the $\mathrm{SU}(2) \times \widetilde{\mathrm{SU}(2)}$ symmetry. For SU(2) one finds

$$
\int \xi_{a}^{* i} g_{i j} \xi_{b}^{j} d^{5} \Omega=\frac{2}{3} N_{5}^{\frac{7}{2}} \pi^{3} \delta_{a b} \kappa_{a} e^{-g-9 p+\frac{x}{2}}\left[2 e^{6 p+2 x}(f-1)+\left(e^{g}+2 e^{6 p+2 x}\right)\right]
$$

with $\kappa_{1}=\kappa_{2}=2$ and $\kappa_{3}=1$, while for $\widetilde{\mathrm{SU}(2)}$ one finds

$$
\int \xi_{a}^{* i} g_{i j} \xi_{b}^{j} d^{5} \Omega=\frac{2}{3} N_{5}^{\frac{7}{2}} \pi^{3} \delta_{a b} \kappa_{a} e^{-g-9 p+\frac{x}{2}}\left(e^{g}+2 e^{6 p+2 x}\right)
$$

with $\kappa_{4}=\kappa_{5}=2$ and $\kappa_{6}=1$. 
Similarly for the one-forms $\mu_{a}^{\xi}$, the scalar products are diagonal. For SU(2) one gets

$$
\int \mu_{a}^{* i} g_{i j} \mu_{b}^{j} d^{5} \Omega=\frac{1}{3} e^{-g-3 p+\frac{x}{2}} \delta_{a b} \kappa_{a} N_{5}^{\frac{7}{2}} \pi^{3}\left[(f-1)\left(a^{2}-1\right)+2 e^{g}\left(2 e^{g}+e^{6 p+2 x}\right)\right]
$$

with $\kappa_{1}=\kappa_{2}=2$ and $\kappa_{3}=1$, while for $\widetilde{\mathrm{SU}(2)}$ one gets

$$
\int \mu_{a}^{* i} g_{i j} \mu_{b}^{j} d^{5} \Omega=\frac{2}{3} N_{5}^{\frac{7}{2}} \pi^{3} e^{\frac{x}{2}-3 p} \delta_{a b} \kappa_{a}\left(2 e^{g}+e^{6 p+2 x}\right)
$$

with $\kappa_{4}=\kappa_{5}=2$ and $\kappa_{6}=1$, exposing the $\mathrm{SU}(2) \times \widetilde{\mathrm{SU}(2)}$ symmetry.

From the above scalar products one can read the gauge kinetic functions for the massless vectors of $\mathrm{SU}(2) \times \widetilde{\mathrm{SU}(2)}$ in 5 -d. For $\mathrm{SU}(2)$ one finds

$$
\begin{aligned}
\mathcal{K}_{\mathrm{SU}(2)}=\frac{1}{2} \mathcal{V}^{-\frac{1}{3}}\left(\|\xi\|^{2}+e^{\phi}\|\mu\|^{2}\right)= & \frac{N_{5}^{\frac{8}{3}}}{12} \pi^{2} \delta_{a b} \kappa_{a} e^{-2 p-g}\left\{(f-1)\left[4 e^{2 x}+e^{\phi}\left(a^{2}-1\right)\right]\right. \\
& \left.+\left[2 e^{g-6 p}+4 e^{2 x}+2 e^{g+\phi}\left(2 e^{g}+e^{6 p+2 x}\right)\right]\right\}
\end{aligned}
$$

where the first term is a contribution from the Einstein-Hilbert term, while the second comes from the $F_{3}^{2}$ in the Type IIB action. ${ }^{6}$ For $\widetilde{\mathrm{SU}(2)}, \mu=e^{-\phi / 2} \xi$ and one simply finds

$$
\mathcal{K} \widetilde{\mathrm{SU}(2)}=\mathcal{V}^{-\frac{1}{3}}\|\xi\|^{2}=\frac{N_{5}^{\frac{8}{3}} \pi^{2}}{3} \delta_{a b} \kappa_{a} e^{-g-8 p}\left(e^{g}+2 e^{6 p+2 x}\right)
$$

The internal volume factor $\mathcal{V}^{-\frac{1}{3}}$ arises from the Weyl scaling of the 10-d metric with pure space-time components so as to have canonical E-H term in 5 -d, i.e. $g_{\mu \nu}^{(10)}=\mathcal{V}^{-\frac{2}{3}}(u) g_{\mu \nu}^{(5)}$.

\section{Massless vectors in MN background}

We will now explicitly apply the above analysis to the case of MN solution for wrapped D5-branes [4]. For simplicity we will focus on the $\widetilde{\mathrm{SU}(2)}$ factor, for which $\mu^{\xi}=e^{-\phi / 2} \xi$.

\subsection{MN Solution}

In MN solution for wrapped D5-branes [4] one has $h_{1}=h_{2}=0$ (no D3-branes $F_{5}=0$, no NS5-branes $\left.H_{3}=0, \chi=0\right)$ and $b=a$. Denoting the radial variable by $u$, the metric reads

$$
d s^{2}=e^{\frac{\phi}{2}}\left[d x^{2}+N_{5}\left\{d u^{2}+e^{2 g}\left(e_{1}^{2}+e_{2}^{2}\right)+\frac{1}{4}\left(\tilde{\omega}_{1}^{2}+\tilde{\omega}_{2}^{2}+\tilde{\omega}_{3}^{2}\right)\right\}\right]
$$

where

$$
\begin{aligned}
e^{-2 \phi} & =\frac{2 e^{g}}{\sinh 2 u}, \quad e^{2 g}=u \operatorname{coth} 2 u-\frac{1}{4}\left(1+a^{2}\right) \\
a & =\frac{2 u}{\sinh 2 u}, \quad f=4 e^{2 g}+a^{2}
\end{aligned}
$$

\footnotetext{
${ }^{6}$ Which is well defined for $A_{4}=0$.
} 
The RR 3-form flux is given by

$$
\begin{aligned}
F_{3}= & \frac{N_{5}}{4}\left\{\tilde{\omega}_{3} \wedge\left[\left(\omega_{1} \wedge \omega_{2}+e_{1} \wedge e_{2}\right)-a(u)\left(\omega_{1} \wedge e_{2}-\omega_{2} \wedge e_{1}\right)\right]\right. \\
& \left.+a^{\prime}(u) d u \wedge\left(\omega_{1} \wedge e_{1}+\omega_{2} \wedge e_{2}\right)\right\}
\end{aligned}
$$

The asymptotic behavior of the radial functions in the UV $(u \rightarrow 0)$ and IR $(u \rightarrow \infty)$ are found to be

$$
\begin{aligned}
a(u \rightarrow 0) & \rightarrow 1-\frac{2}{3} u^{2}, \quad a(u \rightarrow \infty) \rightarrow 0 \\
e^{2 g}(u \rightarrow 0) & \rightarrow u^{2}, \quad e^{2 g}(u \rightarrow \infty) \rightarrow u \\
f(u \rightarrow 0) & \rightarrow 1+\frac{8}{3} u^{2}-\frac{32}{45} u^{4}, \quad f(u \rightarrow \infty) \rightarrow 4 u
\end{aligned}
$$

For later use, notice that

$$
g_{\mu \nu}=e^{\frac{\phi}{2}} \delta_{\mu \nu} \quad, \quad g_{i j}=e^{\frac{\phi}{2}} \hat{g_{i j}} \quad, \quad \operatorname{det} g_{M N}=\frac{N_{5}^{6}}{64} e^{5 \phi} e^{4 g} \sin ^{2} \theta \sin ^{2} \tilde{\theta}
$$

\subsection{Spectrum of massless vector harmonics}

As we have seen, in order to find the spectrum of bound-states that are holographically dual to the massless bulk vectors associated to the three Killing vectors of $\widetilde{\mathrm{SU}(2)}$, one should solve

$$
\frac{1}{\sqrt{g}} \partial_{\mu}\left[\sqrt{g} e^{\phi / 2} f^{\mu \nu}\right]=0
$$

There are two cases to consider $\nu=\hat{\nu}$ and $\nu=u$.

For $\nu=u$ one simply gets

$$
\partial^{\hat{\mu}} f_{\hat{\mu} u}=0 \quad \rightarrow \quad \partial^{\hat{\mu}} \partial_{\hat{\mu}} A_{u}-\partial_{u} \partial^{\hat{\mu}} A_{\hat{\mu}}=0
$$

that allows to express $A_{u}$ in terms of the longitudinal component of $A_{\hat{\mu}}$.

For $\nu=\hat{\nu}$ one gets

$$
\partial_{\hat{\mu}} f^{\hat{\mu} \hat{\nu}}+\frac{1}{\sqrt{g(u)}} \partial_{u}\left[\sqrt{g(u)} e^{\phi / 2} g^{u u} g^{\hat{\nu} \hat{\lambda}}\left(\partial_{u} A_{\hat{\lambda}}-\partial_{\hat{\lambda}} A_{u}\right)\right]=0
$$

Setting $A_{\hat{\mu}}=a_{\hat{\mu}}(u) e^{i p \cdot \hat{x}}$ and $A_{u}=b(u) e^{i p \cdot \hat{x}}$ one can solve for $a_{\hat{\mu}}(u)$ and $b(u)$. Decomposing $a_{\hat{\mu}}(u)$ into longitudinal and transverse components according to

$$
a_{\hat{\mu}}(u)=a_{\hat{\mu}}^{T}(u)+i p_{\hat{\mu}} a_{L}(u)
$$

one finds

$$
b(u)=a_{L}^{\prime}(u)
$$

that can be set to zero by gauge transformations. The surviving transverse components then satisfy an equation

$$
\frac{1}{\sqrt{g(u)}} \partial_{u}\left(\sqrt{g(u)} e^{-\phi / 2} \partial_{u} a_{\hat{\mu}}^{T}\right)-e^{-\phi / 2} p^{2} a_{\hat{\mu}}^{T}=0
$$


that is identical to the equation for a canonical massless scalar $\Phi$. After setting

$$
\Phi=e^{-\phi-g} \mathcal{Y}
$$

the equation is put in canonical form with an effective potential given by

$$
V_{\text {eff }}=\phi^{\prime \prime}+g^{\prime \prime} \approx-\frac{1}{u^{2}} \text { in } \mathrm{UV}
$$

Unfortunately due to the 'pathological' UV behavior there is no spectrum of discrete states associate to the bulk massless vectors. For $\mathrm{SU}(2)$ the story is similar. This behavior is analogous to the one found for the fluctuations of the metric in MN solution [4]. Indeed, the transverse traceless components of the metric fluctuations $h_{i j}^{T T}(u, x)=e_{i j}(p) f_{p}(u) e^{i p x}$ decouple from the rest and satisfy a free massless scalar equation of the form ${ }^{7}$

$$
\left[\partial_{u}^{2}+4 A^{\prime} \partial_{u}-e^{2 A} p^{2}\right] f_{p}(u)=0
$$

For MN solution [4], the relevant equation has been studied in [26, 27] and shown to have a continuous spectrum without a mass gap. Longitudinal and radial components of the metric mix with the active scalars and behave better [26, 27]. Despite the area-law behavior of the Wilson loop in MN background [4], the presence of massless fluctuations casts some shadow on the holographic interpretation as a dual to a confining theory such as $\mathcal{N}=1 \mathrm{SYM}$.

\section{Conclusions and summary}

Let us conclude by summarizing our results and draw lines for future investigation.

We have shown that all RG flows described by the PT ansatz [5] in Type IIB supergravity enjoy exact $\mathrm{SU}(2) \times \widetilde{\mathrm{SU}(2)}$ symmetry in that not only the metric but also background $p$-forms are invariant under diffeomorphisms generated by the six Killing vectors. We have then identified a very general ansatz for the combined fluctuations of metric and $p$-forms that diagonalizes the resulting equations for the bulk massless vectors. Although derived in the context of holography, our ansatz is expected to have much wider applicability in any flux compactification with isometry. Restricting our attention to the case of backgrounds invariant under world-sheet parity $\Omega$, we have illustrated our procedure in the case of MN solution [4] for wrapped D5-branes. The spectrum of massless vector harmonics in this background - very much as the spectrum of massless scalars and transverse traceless fluctuations of the metric $[26,27]$ - is continuous and has no mass gap. This drawback might be related to the impossibility of fully decoupling KK states from the desired physical modes, which survive in the deep IR. In particular the very presence of an exact $\mathrm{SU}(2) \times \widetilde{\mathrm{SU}(2)}$ symmetry is a remnant of the breaking of $\mathcal{N}=4$ to $\mathcal{N}=1^{*}$ with common mass for the three chiral multiplets. Since this symmetry is an exact symmetry of any RG flows described by PT ansatz [5], not excluding KS solution [3], we should conclude

\footnotetext{
${ }^{7}$ The very same equation governs the dynamics of the transverse modes of the supersymmetry partners of the graviton e.g. gravitino, graviphoton, ... as originally shown in [19].
} 
that holographic SYM is still undelivered [44] at least in a top-down approach. The strictly 5-d bottom-up approach embodied by Holographic QCD [37-41] seems more promising in this respect.

\section{Acknowledgments}

We would like to thank C. Bhamidipati, D. Elander, D. Forcella, L. Lopez, J. F. Morales, R. Richter and M. Samsonyan for useful discussions. The work of M. B. was partially supported by the ERC Advanced Grant n.226455 "Superfields" and by the Italian MIUR-PRIN contract 2007-5ATT78 "Symmetries of the Universe and of the Fundamental Interactions". W. dP. acknowledges partial support from the Brazilian agency CAPES and University of Roma "Tor Vergata" for the kindly hospitality. M. B. would like to thank the String Theory group at Queen Mary University of London for the kind hospitality while this work was being completed.

Open Access. This article is distributed under the terms of the Creative Commons Attribution Noncommercial License which permits any noncommercial use, distribution, and reproduction in any medium, provided the original author(s) and source are credited.

\section{References}

[1] S.B. Giddings, S. Kachru and J. Polchinski, Hierarchies from fluxes in string compactifications, Phys. Rev. D 66 (2002) 106006 [hep-th/0105097] [SPIRES].

[2] I.R. Klebanov and A.A. Tseytlin, Gravity Duals of Supersymmetric $\mathrm{SU}(N) \times \mathrm{SU}(N+M)$ Gauge Theories, Nucl. Phys. B 578 (2000) 123 [hep-th/0002159] [SPIRES].

[3] I.R. Klebanov and M.J. Strassler, Supergravity and a confining gauge theory: Duality cascades and $\chi S B$-resolution of naked singularities, JHEP 08 (2000) 052 [hep-th/0007191] [SPIRES].

[4] J.M. Maldacena and C. Núñez, Towards the large- $N$ limit of pure $N=1$ super Yang-Mills, Phys. Rev. Lett. 86 (2001) 588 [hep-th/0008001] [SPIRES].

[5] G. Papadopoulos and A.A. Tseytlin, Complex geometry of conifolds and 5-brane wrapped on 2-sphere, Class. Quant. Grav. 18 (2001) 1333 [hep-th/0012034] [SPIRES].

[6] R. Apreda, F. Bigazzi, A.L. Cotrone, M. Petrini and A. Zaffaroni, Some comments on $N=1$ gauge theories from wrapped branes, Phys. Lett. B 536 (2002) 161 [hep-th/0112236] [SPIRES].

[7] C.P. Herzog, I.R. Klebanov and P. Ouyang, D-branes on the conifold and $N=1$ gauge/gravity dualities, hep-th/0205100 [SPIRES].

[8] R. Apreda, D.E. Crooks, N.J. Evans and M. Petrini, Confinement, glueballs and strings from deformed AdS, JHEP 05 (2004) 065 [hep-th/0308006] [SPIRES].

[9] M. Bianchi, Recent Trends in Superstring Phenomenology, arXiv:0909.1799 [SPIRES].

[10] J.M. Maldacena, The large- $N$ limit of superconformal field theories and supergravity, Adv. Theor. Math. Phys. 2 (1998) 231 [Int. J. Theor. Phys. 38 (1999) 1113] [hep-th/9711200] [SPIRES]. 
[11] S.S. Gubser, I.R. Klebanov and A.M. Polyakov, Gauge theory correlators from non-critical string theory, Phys. Lett. B 428 (1998) 105 [hep-th/9802109] [SPIRES].

[12] E. Witten, Anti-de Sitter space and holography, Adv. Theor. Math. Phys. 2 (1998) 253 [hep-th/9802150] [SPIRES].

[13] For a recent review see e.g. S.S. Gubser and A. Karch, From gauge-string duality to strong interactions: a Pedestrian's Guide, Ann. Rev. Nucl. Part. Sci. 59 (2009) 145 [arXiv:0901.0935] [SPIRES].

[14] S.A. Hartnoll, Lectures on holographic methods for condensed matter physics, Class. Quant. Grav. 26 (2009) 224002 [arXiv:0903.3246] [SPIRES].

[15] S. Bhattacharyya, S. Minwalla and S.R. Wadia, The Incompressible Non-Relativistic Navier-Stokes Equation from Gravity, JHEP 08 (2009) 059 [arXiv:0810.1545] [SPIRES].

[16] T. Hartman, W. Song and A. Strominger, Holographic Derivation of Kerr-Newman Scattering Amplitudes for General Charge and Spin, JHEP 03 (2010) 118 [arXiv:0908.3909] [SPIRES].

[17] J. de Boer, K. Papadodimas and E. Verlinde, Holographic Neutron Stars, arXiv:0907.2695 [SPIRES].

[18] P. Horava, Quantum Gravity at a Lifshitz Point, Phys. Rev. D 79 (2009) 084008 [arXiv: 0901.3775] [SPIRES].

[19] M. Bianchi, O. DeWolfe, D.Z. Freedman and K. Pilch, Anatomy of two holographic renormalization group flows, JHEP 01 (2001) 021 [hep-th/0009156] [SPIRES].

[20] M. Bianchi, D.Z. Freedman and K. Skenderis, How to go with an RG flow, JHEP 08 (2001) 041 [hep-th/0105276] [SPIRES].

[21] M. Bianchi, D.Z. Freedman and K. Skenderis, Holographic Renormalization, Nucl. Phys. B 631 (2002) 159 [hep-th/0112119] [SPIRES].

[22] M. Krasnitz, A two point function in a cascading $N=1$ gauge theory from supergravity, hep-th/0011179 [SPIRES].

[23] M. Krasnitz, Correlation functions in a cascading $N=1$ gauge theory from supergravity, JHEP 12 (2002) 048 [hep-th/0209163] [SPIRES].

[24] A. Dymarsky, D. Melnikov and A. Solovyov, I-odd sector of the Klebanov-Strassler theory, JHEP 05 (09) 105 [arXiv:0810.5666] [SPIRES].

[25] A. Dymarsky and D. Melnikov, Gravity Multiplet on KS and BB Backgrounds, JHEP 05 (08) 035 [arXiv:0710.4517] [SPIRES].

[26] M. Berg, M. Haack and W. Mueck, Bulk dynamics in confining gauge theories, Nucl. Phys. B 736 (2006) 82 [hep-th/0507285] [SPIRES].

[27] M. Berg, M. Haack and W. Mueck, Glueballs vs. gluinoballs: Fluctuation spectra in nonAdS/non-CFT, Nucl. Phys. B 789 (2008) 1 [hep-th/0612224] [SPIRES].

[28] A. Brandhuber and K. Sfetsos, Current correlators in the Coulomb branch of N=4SYM, JHEP 12 (2000) 014 [hep-th/0010048] [SPIRES].

[29] A. Brandhuber and K. Sfetsos, Current correlators and AdS/CFT away from the conformal point, hep-th/0204193 [SPIRES].

[30] S. de Haro, S.N. Solodukhin and K. Skenderis, Holographic reconstruction of spacetime and 
renormalization in the AdS/CFT correspondence, Commun. Math. Phys. 217 (2001) 595 [hep-th/0002230] [SPIRES].

[31] J. Kalkkinen, D. Martelli and W. Mueck, Holographic renormalisation and anomalies, JHEP 04 (2001) 036 [hep-th/0103111] [SPIRES].

[32] D. Martelli and W. Mueck, Holographic renormalization and Ward identities with the Hamilton-Jacobi method, Nucl. Phys. B 654 (2003) 248 [hep-th/0205061] [SPIRES].

[33] M. Bianchi and A. Marchetti, Holographic three-point functions: one step beyond the tradition, Nucl. Phys. B 686 (2004) 261 [hep-th/0302019] [SPIRES].

[34] M. Bianchi, M. Prisco and W. Mueck, New results on holographic three-point functions, JHEP 11 (2003) 052 [hep-th/0310129] [SPIRES].

[35] W. Mueck and M. Prisco, Glueball scattering amplitudes from holography, JHEP 04 (2004) 037 [hep-th/0402068] [SPIRES].

[36] I. Papadimitriou and K. Skenderis, Correlation functions in holographic RG flows, JHEP 10 (2004) 075 [hep-th/0407071] [SPIRES].

[37] J. Erdmenger, N. Evans, I. Kirsch and E. Threlfall, Mesons in Gauge/Gravity Duals - A Review, Eur. Phys. J. A 35 (2008) 81 [arXiv:0711.4467] [SPIRES].

[38] E. Kiritsis, Dissecting the string theory dual of QCD, Fortsch. Phys. 57 (2009) 396 [arXiv:0901.1772] [SPIRES].

[39] U. Gürsoy, E. Kiritsis, L. Mazzanti and F. Nitti, Improved Holographic Yang-Mills at Finite Temperature: Comparison with Data, Nucl. Phys. B 820 (2009) 148 [arXiv:0903.2859] [SPIRES].

[40] W. de Paula, T. Frederico, H. Forkel and M. Beyer, Dynamical AdS/QCD with area-law confinement and linearRegge trajectories, Phys. Rev. D 79 (2009) 075019 [arXiv: 0806.3830] [SPIRES].

[41] W. de Paula and T. Frederico, Unifying Scalars and Light Mesons by Holographic QCD, arXiv: 0908. 4282 [SPIRES].

[42] I.R. Klebanov, P. Ouyang and E. Witten, A gravity dual of the chiral anomaly, Phys. Rev. D 65 (2002) 105007 [hep-th/0202056] [SPIRES].

[43] S.S. Gubser, C.P. Herzog and I.R. Klebanov, Symmetry breaking and axionic strings in the warped deformed conifold, JHEP 09 (2004) 036 [hep-th/0405282] [SPIRES].

[44] C. Csáki, M. Reece and J. Terning, The AdS/QCD Correspondence: Still Undelivered, JHEP 05 (2009) 067 [arXiv:0811.3001] [SPIRES]. 